tained an enormously increased number of primary impulses, so that the emitted waves were more and more continuous, and this method has been followed by that designed by Lepel, in which the arc is replaced by two or more metal disks separated by a minute by two
By substituting a telephone transmitter for the ordinary telegraphic key, it has been found possible to transmit actual speech a distance of thirty to forty miles, while the limit of telegraphy may now be placed at, perhaps, 5,000 miles.

This very brief sketch must necessarily fail to do justice to the manifold improvements that have been introduced into all the details of working, either in spark or arc telegraphy. Each fills at present a definite sphere, and we have by no means arrived at finality in this interesting branch of the earliest type of elec trical engineering.

\title{
HIGH AND LOW WATER ALARMS FOR BOILERS."
}

\section{NGENIOUS SAFETY DEVICES.}

\author{
B Y W. H. WA K EM A N.
}

FIG. 1 illustrates the first boiler that I ever had charge of, and the first low-water alarm that filled me with consternation. The former is still known as the locomotive boiler, but the latter has long since passed into oblivion, or at least into disuse, so far as general practice is concern was secured in 1875 , and the low-water alarm was located as shown in compliance with a city ordinance, which stated that all boilers used in that city should be equipped with this safety device as a positive preventer of boiler explosion. Previous to the enactment of this law, a boiler located near the center of

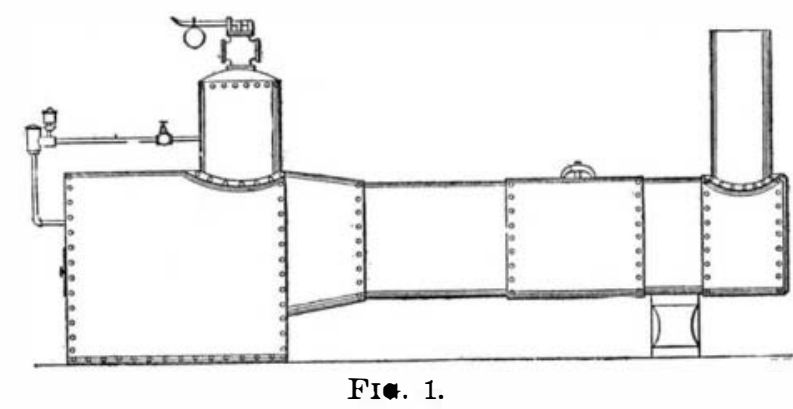

the city in question had exploded and caused the death of twenty-two persons, and the only cause given by those immediately concerned in the catastrophe, and believed by the general public, was lack of water sufficient to cover the plates exposed to the action of heat.

When viewed in the light of our present knowledge of the subject, it seems at least strange that anybody could believe that there was only one cause for boiler explosions, but for several years after this first experience I heard no other cause mentioned, and it was by reports published by the Hartford Steam Boiler inspection and Insurance Company that the many inspection and Insurance Company that the many real causes for these destructive failures were made
plain.

I very naturally supposed that if the neglect of one man caused the loss of so many lives and the destruction of thousands of dollars worth of property, he would at least be discharged in disgrace, and never allowed to have charge of a steam boiler again; hence I was greatly surprised to find that the engineer in charge of the exploded boiler, who was also the fireman and water tender, was still in charge of the machinery in that plant after the wreckage was cleared

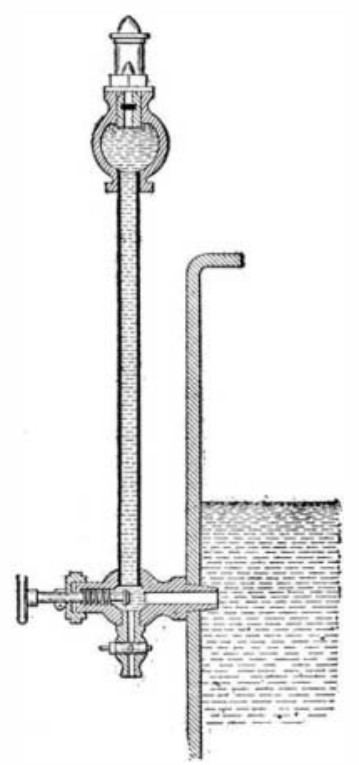

FI. 2.

away and a new boiler set. The reason given by the owner for this action was that nobody could have prevented what they called an accident, hence their engineer was not to blame. This was not due to any particular regard for the engineer or his abilities, but to save the expenses of paying damages to the relatives ar d dependents of those who were killed, and directly to those who were injured.
The main part of this low-water alarm is attached to the front head about 3 inches above the crown sheet, for a small boiler, while a smaller pipe is tapped into the dome, in order to secure dry steam. A power pump was installed for the purpose of drawing water from a driven well and forcing it into this boiler, but it was almost impossible to bring water up to this pump because the strainer offered much resistance to the flow of water, and pressure of the atmosphere cannot act directly on the surface of water outside of such a well and thus force it up into the pipe. This pump was driven by a pulley on the crank shaft, and it was customary to remove the governor belt and run the engine as fast as was safe, until water appeared at the pump, after which the governor belt was replaced and water was assured for the remainder of that day.

When this boiler was installed, it was filled with water to the second gage by hand, a fire was built in the furnace, and efforts made to secure water by the pump, which were not successful the first day. While we were working on the pump the water level was lowered until it left the front-head connection uncov. ered. Steam was thus allowed to enter this pipe and passing upward melted the soft metal with which the passing upward melted the soft metal with which the
head of the "device was filled. This action released the whistle valve and in an instant a shrill blast startled all who were near. Somebody opened the safety valve, and somebody else drew out the fire in record time. Of course, the safety valve ought not to have been opened, but our experience was limited at that time. As the metal which prevented the whistle from blowing under normal conditions the whistle from blowing under normal conditions
was gone, it was necessary to remove the device and have it repaired before it could be used again. The objection to this was the delay caused, and the expense incurred. On the other hand, it was a valuable feature, because it assisted materially in causing us to be more careful. However, as no more boilers exploded, the excitement subsided, the ordinance was repealed, and the use of high and low-water alarms is now optional with all steam users in that city.

Fig. 2 illustrates a similar device except that only one connection is required, and an angle valve is provided for shutting off the alarm whenever it needs repairs. The objection to the presence of this valve is that it may be shut when it ought to be open, hence the fireman or water tender may allow the water level to fall lower than it ought to, and no alarm will be to fall lower than it ought to, and no alarm will be given. However, it should be considered a necessity, and suitable measures taken to prevent unauthorized
persons from meddling with it. If a water tender persons from meddling with it. If a water tender
closes it to prevent an alarm from being given on account of carelessness and neglect of duty on his

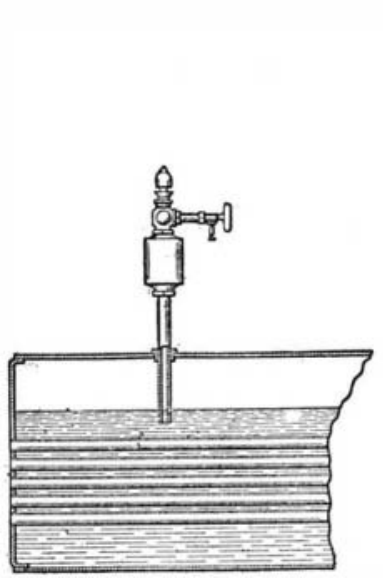

FIG. 3.

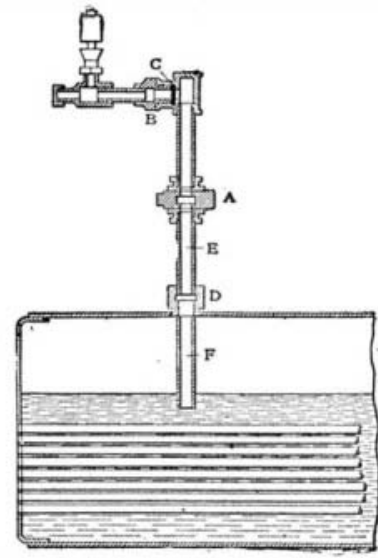

FIG. 4. part, he should be reprimanded, and if the offense is repeated he ought to be discharged.

This low-water alarm operates as follows: As long as the inlet is covered by water, the vertical pipe remains cool, as there is no circulation through it, but if the water level falls low enough to uncover the inlet, steam enters the pipe and, ascending to the top, melts the soft-metal plug which covers the whistle inlet, as it is made of an alloy that melts at
212 deg. F. Having melted the plug, steam blows freely through the whistle, giving an alarm with no uncertain sound.

In order to restore normal conditions, shut the angle valve to stop the noise, and open the drip cock to let

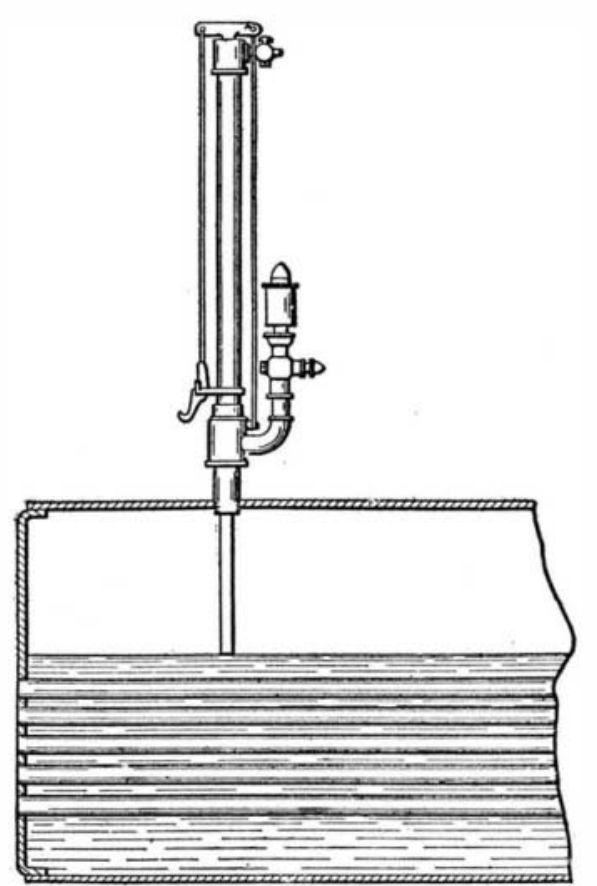

FIG. 5.

out the water standing in the pipe. Pump water into the boiler until the alarm-pipe inlet is covered, and do it without delay, for although the front-head connection shown is, or ought to be, set high enough to blow the whistle before the upper tubes in a tubular boiler or the crown sheet of a locomotive boiler are damaged by heat, or are liable to be warped by the introduction of more water, still the water level will soon become too low for safety, especially as the alarm is sure to distract attention from everything else.

Unscrew the whistle, put in another plug, screw the whistle down hard on it to make a tight joint, then admit water to pipe slowly (after closing the drip

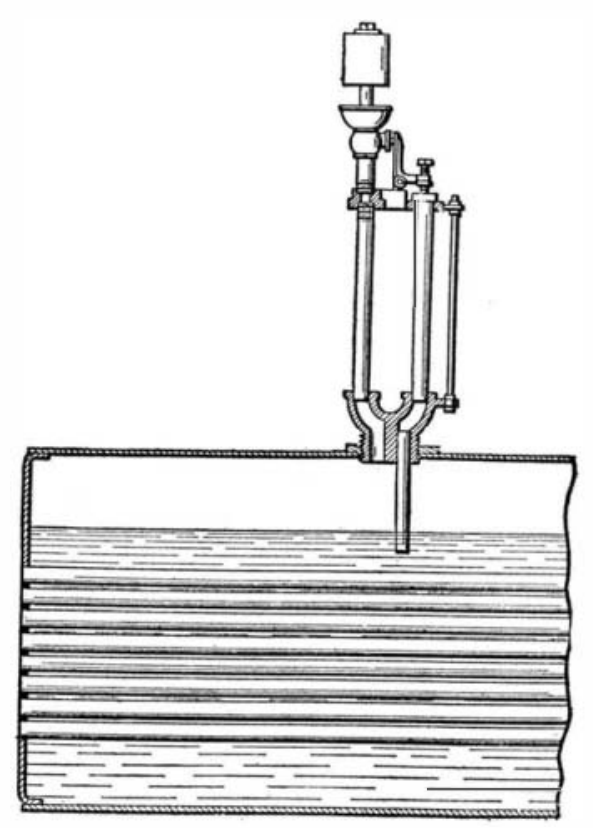

FIG. 6.

cock), for unless this is done cautiously the new plug will be melted by heat, because the temperature of water in an ordinary boiler used for power purposes is much above $212 \mathrm{deg}$. This àlarm can be attached to the shell of a vertical boiler, where it will be equally effective.

The following alloy will melt at about 212 deg. F.: Lead, 1 part; tin, 3 parts; bismuth, 5 parts. Jt is claimed that any alloy of which bismuth forms a 
part is not reliable for this service, because it will not retain its original nature indefinitely, hence will fail to melt when it ought. to. It is quite possible that this refers to regular fusible plugs as used in steam boilers, as they are exposed to very hot wate on one side, and the temperature of a fire on the other, hence pure Banca tin is recommended for them; but this metal is useless for the low-water alarms illustrated in this article, because it requires a temperature

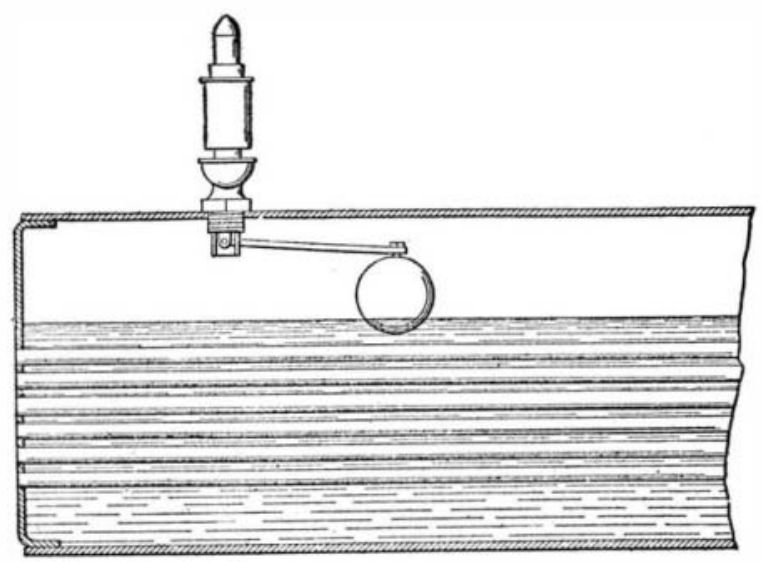

Fi*. 7.

of $445 \mathrm{deg}$. to melt or fuse it. Of course these fusible plugs are exposed to a much higher temperature on one side only, as the pressure of steam must be raise to 400 pounds absolute in order to secure this temperature, and that is seldom or never realized, eve in these days of high pressure. The fire does no melt them because heat passes rapidly into the water, but steam at the same temperature has a very different $\epsilon$ ffect, causing the plugs to melt.

Fig. 3 illustrates another device for giving an alarm when the water level is approaching the danger line It is attached to the shell, but projects down through it far enough to submerge the lower end when th water level is right for working conditions, but when it falls to a depth of about 4 inches over the uppe tubes, or flues, as the case may be, steam enters the vertical pipe and, melting a soft-metal plug in the body of the device, causes the whistle to blow.

When this happens, shut the valve, pump in more water, remove the whistle, put in another plug, replace the whistle and open the valve slowly. Care must be taken to make tight joints, for even a slight leak will cause a circulation that may raise the temperature high enough to melt the new plug when there is plenty of water in the boiler.

Superficial examination of this device, in connection with the knowledge that water and steam in a boiler are of the same temperature, will cause a fireman to wonder why the alarm operates as described. This point is made clear when he remembers that under working conditions water will not circulate throug this vertical pipe, but will go up into it and stay there until it becomes comparatively cool, provided every joint is absolutely tight. If the water level falls low enough to allow steam to enter the end of this pipe, conditions are at once changed. The full temperature due to the pressure carried is transmitted to the plug, and when. condensation due to radiation results in the appearance of water at this point, it runs back into the boiler and is replaced by more steam until the plug melts.

On a certain occasion I stated that water and steam in a boiler were of the same temperature (as herein mentioned), and was promptly corrected by a listener, who stated that he pumped cold water into his boiler and the plates around the feed pipe where it was and the plates around the feed pipe where it was
tapped into the boiler were cool, hence water could



Fi. 8

not be as hot under these conditions as the steam above it. So far as his limited comprehension of th subject would admit, he was correct, but in all such cases it is expected that common sense will be used and intelligently applied. Of course it is generally understood that when the foregoing statement is made it refers to water that has been in a boiler long enough to attain its highest possible temperature; also, that the boiler is designed so that circulation is at least nearly perfect. Although water has the same tem perature under these conditions, it does not flash into steam, because it lacks the latent heat required by the process of generating steam.

Fig. 4 illustrates another low-water alarm in which a soft-metal plug is an important feature. It is attached to the shell and extends down through it to the low-water level, wherever that may be. While this depends on the judgment of the engineer in charge, after taking all conditions into consideration, there are few cases in which the water should be less than 4 inches deep over the tubes of a tubular boiler, or the crown sheet of the locomotive type, when the whistle blows, and 6 inches is better in many cases.

When the whistle in Fig. 4 blows for low water, the plug cock $A$ must be closed and more water pumped into the boiler. Unscrew the cap $B$, remove what remains of the plug $C$, insert a new one, screw on the cap until it is firmly seated, and open $A$ slowly. If a cap until it is firmly seated, and open $A$ slowly. If a
globe valve is used here, there will be less danger of globe valve is used here, there will be less danger of
its sticking fast when it ought to work freely, and it can be repaired with little trouble and small expense. A special bushing is shown at $D$, into one end of which the pipe $\boldsymbol{E}$ is connected, while $\boldsymbol{F}$ is screwed into the opposite end, leaving a small space between them. Care should be taken when making up such a fitting to know that the pipes enter far enough to secure a firm hold, also that there is an open space between them as illustrated. The shells of many boilers are only $3 / \%$ inch thick, while some are less therefore, a first-class job cannot be secured by screwing in the bushing from the outside, as there are not enough threads available. By starting the tap from the outside, and finishing it from the inside, it is practicable to insert the bushing from the inside and thus prevent it from blowing out, even if the threads are corroded by long service until they no longer are strong enough to resist the pressure put upon them.

An objection to all kinds of low-water alarms which depend on the melting of a plug for their operation is that they cannot be tested in service, therefore it is impossible to know their true condition. Of course

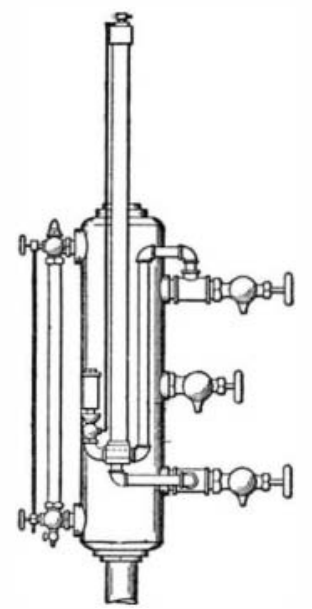

Fi*. 9.

it is possible to let the water level fall until the whistle does, or ought to, blow, and observe the result, but in either case it becomes necessary to insert a new plug that has not been tried, thus restoring the uncertain element. The following plan is recommended for such cases: Secure a dozen plugs made from the sam alloy. Insert one and test it by lowering the water level until steam enters the pipe. If the plug melts promptly, it is good evidence that they are reliable under given conditions for one year, hence a new one under given conditions for one year, hence a new one
should be inserted annually. The time may be shortened if greater safety is demanded.

Fig. 5 illustrates a device for blowing a whistle when the water level falls too low for safety, the operation of which is based on the principle that brass and iron do not expand equally when heat is applied. Under normal conditions the lower end of the vertical pipe is submerged in water. As soon as the water level. is lowered until it begins to assums the position shown in the illustration, all water runs out of the pipe and is replaced by steam, but it is not admitted to the whistle by this action. The temperature of the pipe, which is made of brass, is raised and it is lengthened accordingly.

The rods on either side of the pipe are made of iron, but inasmuch as the coefficient of expansion for brass is about 50 per cent greater than for iron, the pipe expands more than the rods for an equal rise in pipe expands more than the rods for an equal rise in temperature, and it is quite possible that the latter
are not as hot as the former while in service, because are not as hot as the former while in service, because
steam does not come into direct contact with them, steam does not come into direct contact with them
hence the actual difference in expansion is greater than hence the actual differe
the foregoing implies.

As the rods are anchored at their lower ends, the difference in length causes the whistle to open and admit dry steam from the upper part of the steam space, thus giving the alarm in time to prevent damage from low water. When the usual water level is restored water fills the pipe, again causing it to contract and close the whistle valve. This alarm can be tested by opening the pet cock shown at the top and allowing hot water to circulate through the pipe, thus raising its temperature the same as if steam were admitted to it, without lowering the water level.

In case the whistle does not blow when this test is applied, the rods should be shortened by nuts provided for this purpose, until the valve is barely open. If the whistle continues to blow after the standpipe

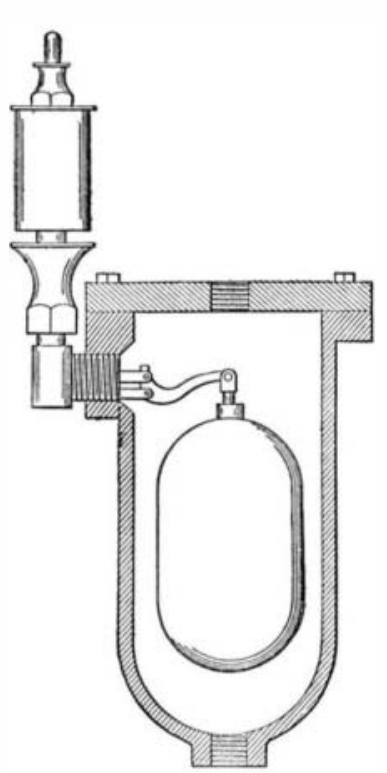

Fiศ. 10.

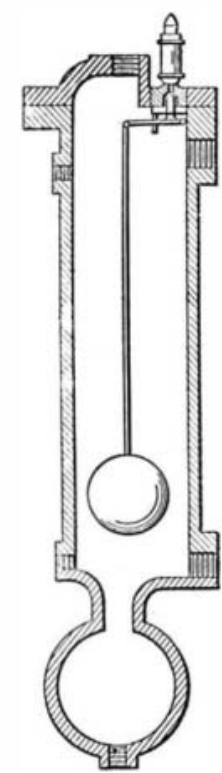

Fia. 11. becomes cool, the rods must be lengthened by adjusting the nuts in the opposite direction.

Fig. 6 illustrates another low-water alarm which is operated by the expansion of a brass pipe, but the laim that is plainly made for it to the effect that it depends on the difference in the expansion of the two metals is apparently not warranted. The claim is as follows: The left-hand vertical pipe is made of wrought iron, and as it is connected directly into the steam space of the boiler, it is always full of dry steam up to the valve just below the whistle. The right-hand pipe extends down below the normal water level, hence is filled with water under working conditions and usually is comparatively cool because there is no circulation through it, as it forms. what is known in hydraulic work as a "dead end."

This pipe is made of brass and terminates in a cap on which rests an adjustable screw carried by the outer end of a bell-crank lever. The upper end of this lever rests against the stem of the whistle valve, and the screw is adjusted so that when the pipe below it is cool, the lever touches the whistle valve lightly. When this pipe is heated by steam on account of lowering the water level, it expands, raises the horizontal end of the lever, causing the vertical end to open the whistle valve and sound an alarm that continues until the water level is raised, as no valve is provided for shutting it off by hand.

This alarm can be adjusted while pressure is on the boiler, hence the left-hand pipe is then as hot as steam can make it: consequently, it makes no difference whether it is made of iron or brass, because its length when expanded becomes the standard regardless of the amount of expansion. The right-hand pipe must be filled with cool water when the adjustment is made, and as soon as it is expanded by steam the whistle

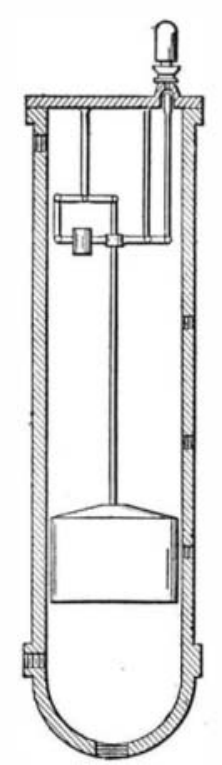

Fiศ. 12.

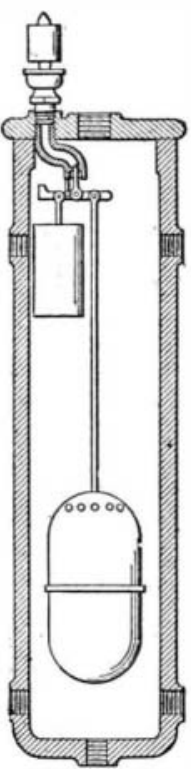

FI*. 13.

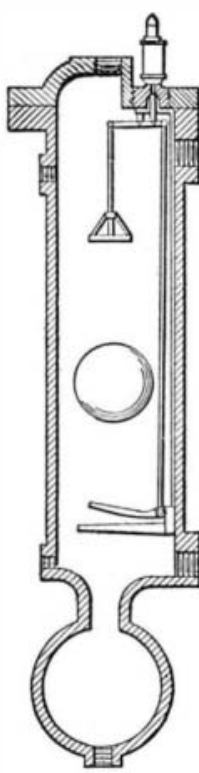

F1\%. 14. blows (if a correct adjustment has been made) regardless of the length of the left-hand pipe, hence it is plain that the difference in expansion has no bearing on the device or its operation. A reinforcing ring is riveted to the shell in order to afford a more secure fastening for the alarm, and this is worthy of adoption in all such case

One advantage of low-water alarms operated by the 
expansion of metal is that they can be tested at any time and their condition proved beyond dispute. The only disadvantage is that they require a fine adjustment, which is too difficult a task for the ordinary fireman or water tender to complete and maintain but, on the other hand, there seems to be no good reason why the engineer should not attend to this exclusively, and thus avoid all trouble along this line. The low-water alarm illustrated in Fig. 7 consis The low-water alarm illustrated in Fig. 7 consists
of a whistle screwed into the shell, containing a lever of a whistle screwed into the shell, containing a lever
valve operated by a float which rises and falls with the valve operated by a float which rises and falls with the
water level. This arrangement will undoubtedly work well as long as the float remains in good order, but unless it is of superior design and made in a more workmanlike manner than many of those found in service, it will prove a source of more worry and vexation than of comfort.

A dishonest boiler attendant cannot tamper with this low-water alarm while it is in service, as the float is in a secure place, and there is no globe valve on the outside to close, in order to prevent exposure
of carelessness in allowing the water level to fall too low for safety.

The disadvantages of this device are that if the float collapses or fills with water and sinks while there is still plenty of water in the boiler, the whistle will blow and no legitimate way of stopping it is provided. Such defects, or a failure of the lever mechanism. Such defects, or a failure of the lever mechanism,
cannot be repaired easily, as the boiler must be laid off for this purpose, and if this is not practicable, the low-water alarm must be dispensed with until an opportunity arrives for entering the boiler and making necessary repairs.

Fig. 8 shows what may be called a "home-made" lowwater indicator. It consists of a block of wood floating on the water to which is attached a brass rod carried through a stuffing box in the shell. A stout cord or an annealed wire is carried from this over two pulleys and supports a weight suspended in front of a dial, and the position of this weight indicates the water level. This device was describe

Fig. 9 illustrates a low-water alarm that is not Fig. 9 illustrates a low-water alarm that is not
attached directly to the boiler but to the water column. The. lower connection is made by removing the first gage cock and screwing in a close nipple followed by a tee, the outer end of which receives the gage cock for use as before. Into the side outlet of this tee a close nipple is screwed, followed by an ell and other connections as shown. The upper connection is made by removing the third gage cock and proceeding as before, using brass pipe and fittings because they rust and corrode less than iron.

While there is one gage, or more, of water in the boiler, the standpipe remains full of comparatively cool water, but when the water level falls until there is only about one inch left in the glass, steam enters the standpipe and as it is made of copper it lengthens, thus opening the whistle valve by means of the rod shown, and admitting steam from the third gage cock through a cross passage to the whistle, although water through a cross passage to the whistle, although water
comes first, as a small amount always stands in the ertical pipe which stands parallel to the standpipe. Fig. 10 is an independent water column to be used as a low-water alarm. It contains a steel float to secure the required strength and it is coated with copper to prevent corrosion. A water gage and three gage cocks can be tapped into this column if desired. water enough to keep. the float in its highest position, the whistle is silent, but when it falls and lowers the float, the alarm is given with no uncertain sound. It can be tested at any time by opening the drip valve. The whistle and the float are connected to the body of the water column, hence when repairs are necessary the cap screws shown are removed and a union in the pipe below is disconnected, thus leaving the column packing an be used several times if one side of it is coated with graphite. After repairs are completed the coated with graphite. After repairs are completed the
column can be returned without disturbing the piping. column can be returned without disturbing the piping.
The cap of this column is flat, therefore any ordinary wrench can be used to tighten the cap screws without inconvenience, which is a valuable feature. Special care should be taken when installing this or any other water column to set it high enough to give an alarm when there is at least 4 inches above the tubes, flues or crown sheet.

Fig. 11 resembles the preceding, but is much longer hence is suitable for boilers in which the water leve may vary to a greater extent without causing damage, as the extreme length of this column is nearly 5
feet. The float is in the form of a sphere, which is the strongest that can be devised. In this case the whistle and its controlling mechanism are attached to the cap, hence when this is removed all the working parts come out with it. This is a very convenient plan when it is practicable to run the pipe connection directly into the body, as shown by the larger tapped holes, but there are many cases in which it is better to make connections into the top and bottom, thus making a union is used in the piping, which would otherwise be unnecessary.

Fig. 12 illustrates another low-water alarm in which the controlling mechanism is attached to the cap. This does not include a float, hence there is no danger of its collapsing or filling with water. One large gravity weight on the end of a rod and a small solid weight on one of the compound levers operate to open and close the whistle valve, according to the height of
water in the boiler and hence in the column. When there are two gages of water, the larger weight so nearly floats that the small one is sufficient to counternearly floats that the small one is sufficient to counter-
balance it and keep the whistle valve closed, but when the water level falls, leaving one free, it opens the whistle valve and sounds an alarm.

Fig. 13 is similar to the foregoing, but the compound levers are dispensed with, and only one used instead, consequently the upper solid weight is larger, but the Many boilers have been seriously damaged, some of them beyond repair, by allowing water to get low in them beyond repair, by allowing water to get low in
them with a brisk fire in the furnace, and others have suffered from the effects of fire under them before they were filled at all, yet only a comparatively few explosions have occurred directly from this cause, and there is no mystery about any of them. If a plate or a crown sheet is red hot from lack of water, its original strength is greatly reduced, hence the ordinary working pressure may cause it to fail, and it may not have sufficient strength to destroy much property, for it is plain that a weak boiler will rupture before a high pressure can be secured, hence the resulting damage will be light.

Furthermore, the overheated part may fail just as more water is pumped in, but it is doubtful if the entrance of water into an overheated boiler has ever caused the instantaneous increase of pressure with which it is charged, or resulted in the formation of a mysterious gas that could not be controlled. Experiments that have been made under working conditions to prove the truth of claims made for the aforementioned destructive agents have failed to show that we are in danger from them.

There can be no doubt about the truth of claims expensive delays to repair bills, damaged boilers and keep boilers properly supplied with water, and these are sufficient to warrant every precaution that can be taken to prevent such accidents without causing them to appear mysterious, or uncontrollable. The variety of low-water alarms already described and illustrated in these pages, and the large numbers of them sold and put into service on various ki

Previous illustrations are of devices that guard against low water only, but very expensive accidents have happened on account of high water in boilers, because under such conditions there is danger of delivering hot water to the engine instead of steam, and as they are not built for such abuse the weakest parts must fail, and although such accidents seldom result in loss of life, they destroy valuable machinery and cause delays to business.

Fig. 14 illustrates a water column fitted with an alarm for both high and low water, which operates as follows: A spherical, seamless float without means for attaching a lever or rod to it is put into the column which is then partly filled with water to secure working conditions. The float stays on the surface or is only partly submerged, bringing it into the position shown. The upper weight is suficient to keep the whistle valve closed, but if the water level is raised cor than good practice admits, the float comes in contact with the upper weight, raises it and blows the whistle. If the supply of water to the boiler is shut off and the process of evaporation is continued, the float is lowered with the water level until it rests on the lower lever shown, and this action blows the whistle. As the float is not fastened to anything, it is free to turn in every direction, hence it cannot durable in practice. All of the mechanism is fastened to the cap, hence when this is removed the internal to the cap, hence when
parts come out with it.

\section{THE USE OF CELLULOID IN} MICROSCOPY.

In making micro-chemical analysis, says Prof Sommerfeld in Die Gummi-Industrie, it is often necessary to use fluoric acid, fluorammonium and other substances which attack glass; the object plates are thereby rendered "matt," like ordinary ground glass, so that it is desirable to use, instead of glass, some othe material which is not so attacked-and of these celluloid is the best. There is another reason for this, that the reagents which attack glass are often used to show micro-chemically the presence of elements which are present in glass, as for instance soda, potash, or lime In such cases, glass is impossible to be used, as when it is in the least attacked by a reagent, the chemist is uncertain whether the soda, potash or lime come from the material being tested, or from the glass. Celluloid is suited to such work by reason of its resist ance to reagents; but cannot be used in such cases, when alkaline reagents are used, as these attack celluloid. In such cases, therefore, glass must be used. A second advantage in the use of celluloid in microscopy is as follows: For many purposes it is desirable to have object plates which are perforated like a sieve; this is more readily attained with celluloid than with glass. The perforated plates are very desirable in many cases of measurements of thin sections in microscopy and photography. The thickness of the thin sections cannot be measured with the desired accuracy when below the sections to bə measured or even over them glass or other transparent material is cemented. But for mineral sections, even in very limited areas not covered or underlaid by the transparent plate, accurate optical interference methods may be employed to determine the thickness.

If there is only one mineral to be measured, one hole in the object plate would be enough; but often there are several places in the section to be measured, as in petrography, there is usually a number of very minute crystals, so that the advantage of a perforated object plate is apparent, and this is possible only with celluloid.

Incidentally, it may be remarked that mica or gypsum, which the layman might consider as suitable for such purposes, is perfectly useless for mineralogical or micro-chemical work; for this one can use only amorphous materials; as crystalline substances, such as mica and gypsum, counteract the interference tests shown by polarized light.

The employment of sieve-like perforated plates is also to be recommended for micro-chemical analysis In making permanent preparation, the precipitates which are formed at once by the addition of the reagent, must often be cemented by Canada balsam or similar material between the two glass plates of the slide. The watery solution from which the characteristic precipitate has been formed must be removed; but this may not be effected by evaporation, as the soluble this may not be effected by evaporation, as the soluble
salts in the solution would crystallize and cover the precipitate. It is, however, very easy to remove the solution when the slide is perforated. At first one must place thereunder a non-perforated slide; and when the precipitate is formed this is to be carefully removed; which will permit the greater part of the solution to drop through, taking with it, it is true, a very slight portion of the precipitate; but enough will remain for all practical purposes.

It may further be mentioned that celluloid is, in many cases, cheaper than glass for slide-covers; and that it has only one quality which acts against its use in microscopy-its former habit of getting cloudywhich nowadays is seldom the case. If it were possible to make a perfectly incombustible celluloid-which many an inventor has considered that he has produced

-this material would have in microscopy the further advantage, that it would permit cementing the object to it by heating Canada balsam. At present, however where celluloid is to be used, one must either use a solution of this material or employ another substance. As yet, the employment, of celluloid for lenses is not successful.

\section{"MITOHINETISM."}

A $\mathrm{T}$ a joint meeting of the Botany and Zoology sections of the British Association for the Advancement of Science, Prof. M. Hartog described "Mitokinetism, the new force." His paper was welcomed heartily as an interesting and valuable contribution to the Confer ence. Prof. Hartog explained that by a new force $\mathrm{b}$ meant one of which the character and distinctness had
only newly been recognized. The force was discovered in 1873 or thereabouts; but its distinctness was only finally demonstrated by the observations of Pentimalli in 1909 and the author's own interpretation of these results in the present year. When the cell divides, a curious figure is formed, like a spindle with (in animals) a sphere at either end, from which rays diverge outward. The inner rays are concave to the axis of the spindle, a point which is very important. Such a figure can only be produced by a force whose intensity decreases as the square of the distance (a Newtonian force), and one which has two opposite manifestations, such as source and sink, heat source manifestations, such as source and sink, heat source
and refrigerator, positive and negative (electrostatic), north and south (magnetic). Such forces are called "dual forces." Prof. Hartog showed that none of the recognized dual forces could be invoked, and that the name "Mitokinetism," which he had given some years ago to avoid any prejudgmert of its nature, must now be accepted as that of a new force, unknown outsids 